\title{
Revelation of Diabetics by Inadequate Balanced SVM
}

\author{
Dr G Revathy a, Dr R.Madonna Arieth ${ }^{\mathrm{b}}$, Mrs D.Kalaiabiramic ${ }^{\text {, }}$ and Mrs R.Aruna ${ }^{\mathrm{d}}$ \\ a \\ Assistant Professor in Department of CSE, Erode Sengunthar Engineering \\ College(Autonomous), Perundurai, Erode, Tamilnadu \\ bAssociate Professor in Department of CSE, Sri Venkateshwara College of Engineering and Technology, Chittoor, AP. \\ ${ }^{\mathbf{c}}$ Assistant Professor in Department of CSE, Vivekanandha College of Engineering \\ for Women, Thiruchengode, Tamilnadu \\ dAssistant Professor in Department of IT, Kongunadu college of Engineering and Technology, Trichy, Tamilnadu
}

Article History: Received: 11 January 2021; Accepted: 27 February 2021; Published online: 5 April 2021

\begin{abstract}
Diabetic Irish (DI) is human eye disease among people with diabetics which causes damage to irish of eye and may eventually lead to complete blindness. Detection of diabetic Irish in early stage is essential to avoid complete blindness. It will give us accuracy of which algorithm will be suitable and more accurate for prediction of the disease. To maintain more accuracy we follow feature extraction using SVM which gives $85 \%$ accuracy results compared with previous techniques.
\end{abstract}

Keywords: K-Nearest Neighbor, Random Forest, Support Vector Machine and Neural Networks.

\section{Introduction}

Diabetes is persistent and limb syndromes that transpire when the pancreas does not ooze enough insulin or the body is incapable to progression it accurately. Over time, diabetes distress the circular system, including that of the iris. Diabetes Iris (DI) is a therapeutic stipulation where the iris is scratched because of fluid seep out from blood vessels into the iris. It is solitarily the majority familiar diabetic eye diseases and a foremost cause of blindness. Nearly 415 million diabetic patients are at menace of having blindness because of diabetics. It transpire when diabetes damages the tiny blood vessels inside the iris, the light sensitive tissue at the back of the eye. This tiny blood vessel will leak blood and fluid on the iris forms features such as micro-aneurysms, hemorrhage, hard exudates, cotton wool spots or venous loops. Diabetic Irish can be confidential as non-proliferative diabetic Iris (NPDR) and proliferative diabetic Iris (PDR). Depending on the existence of features on the iris, the stages of DR can be notorious. In the NPDR stage, the disease can progress from mild, restrained to severe stage with an assortment of echelon of features except less augmentation of new blood vessels. PDR is the sophisticated stage where the fluids sent by the iris for sustenance trigger the growth of new blood vessels. They grow along the iris and over the surface of the clear, vitreous gel that fills the inside of the eye. If they leak blood, severe vision loss and even blindness can result. At the moment, perceive DI is a protracted and instruction manual process that necessitate a qualified clinician to scrutinize and appraise digital color photographs of the iris. By the time human readers propose their reviews, often a day or two later, the deferred results lead to lost follow up, miscommunication, and deferred treatment.

\section{Work Flow}

The problem arised is that the currently, detecting DI is a time-consuming and manual process that requires a trained clinician to examine and evaluate digital colour photographs of the iris. By the time human readers submit their reviews, often a day or two later, the delayed results lead to lost follow up, miscommunication, and delayed treatment. Diabetes Iris (DI) is a medical condition where the iris is damaged because of fluid leaks from blood vessels into the iris. It is one of the most common diabetic eye diseases and a leading cause of blindness.

Nearly 415 million diabetic patients are at risk of having blindness because of diabetics. It occurs when diabetes damages the tiny blood vessels inside the iris, the light sensitive tissue at the back of the eye. so we collect the datasets of eye.After that the dataset is divided into two sets, one for training where most of the data is used and the other one is testing. In training set four different classification algorithms has been fitted for the analysis performance of the model.

The algorithms we used are k-Nearest Neighbour, support vector machine and neural networks. After the system has done learning from training datasets, newer data is provided without outputs. The final model generates the output using the knowledge it gained from the data on which it was trained. In final phase we get the accuracy result.

\subsection{Iris Image Acquistion}

Initially the eye image is captured with the help of certain cameras, and stored in the database which contains normal as well as abnormal results of iris. Captured eye image looks as shown in fig. 


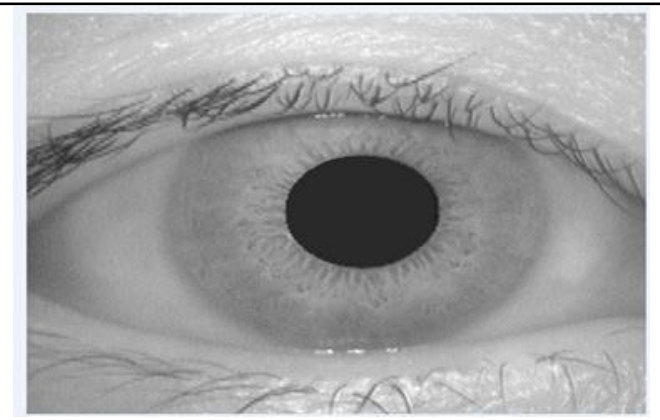

\subsection{Preprocessing}

The preprocessing is done in order to reduce the presence of noise in the iris image and enhancement is done in order to manipulate an image so that the result is more suitable than the original.

It makes the hidden features of an image more available for us. Enhancement is done for improving the details of an image.

It has four steps as:

1. Convert the image to RGB TO HSI.

2. Apply median filtering.

3. Use the CLAHE method (Contrast -limited adaptive histogram equalization)

4. Convert the image to HSI TO RGB.

STEP 1- CONVERT RGB TO HSI IMAGE: luminance.

RGB are all co-related to the colour intensity (luminance). We cannot separate colour information from

HSI used to separate image luminance from colour information. It is make easier when we working on luminance of image.

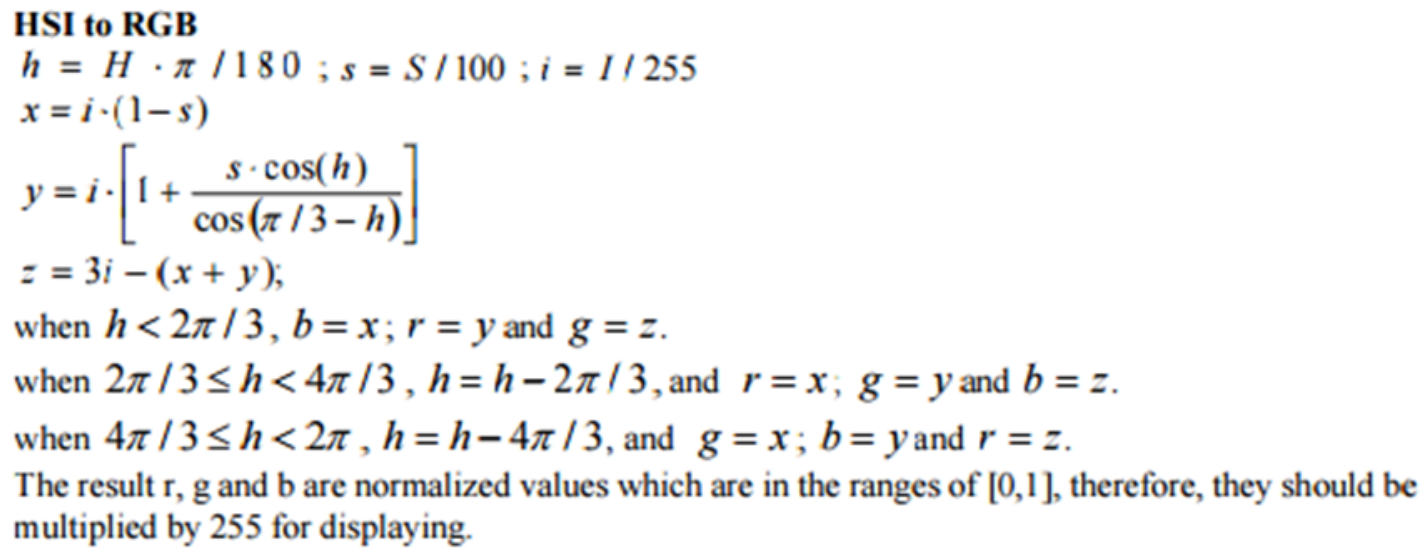

\section{STEP 2 - MEDIAN FILTERING:}

Median filtering works by moving through the image pixel by pixel, replacing each value with the median value is to improving the result.

Median filtering is a non linear digital filtering technique, often used to remove noise from image or signal. Such noise reduction is a typical pre-processing step to improve the result of later pre-processing. For example edge detection of an image. Median filtering is one kind of smoothing technique, as is linear Gaussian filtering. All smoothing techniques are effective at removing noise in smooth patches or smooth regions of a signal, but adversely affect edges. Often though, at the same time as reducing the noise in a signal, it is important to preserve the edges. Edges are of critical importance to the visual appearance of images.

STEP 3 - CLAHE (Contrast - limited adaptive histogram equalization)

It is technique to enhance the visibility of local details of image by increasing the contrast of image. Adaptive histogram equalization (AHE) improves on this by transforming each pixel with a transformation function derived from a neighbourhood region. It was first developed for use in aircraft cockpit displays. In its simplest form, each pixel is transformed based on the histogram of a square surrounding the pixel, as in the figure below. The derivation of the transformation functions from the histograms is exactly the same as for ordinary histogram equalization. The transformation function is proportional to the cumulative distribution function (CDF) of pixel values in the neighbourhood. Pixels near the image boundary have to be treated specially, because their neighbourhood would not lie completely within the image. This applies for example to the pixels to the left or above the blue pixel in the figure. This can be solved by extending the image by mirroring pixel lines and columns with respect to the image boundary. Simply copying the pixel lines on the border is not appropriate, as it would lead to a highly peaked neighbourhood histogram. 


\subsection{Image Segmentation:}

Segmentation is done in order to find inner and outer boundaries of the iris. By subtracting pupil from sclera, we will get the iris part of an eye. Once the iris region is segmented from an eye, the next step is to transform the iris region into fixed dimensions. After subtraction, we will get the iris pattern into circular shape.

Image segmentation is a technique and process which divide the image into different feature of region and extract out the interested target. Here features can be pixel gray scale, colour, texture, etc. Image segmentation plays a fundamental role in many computer vision applications. In pattern recognition approaches image segmentation enables the isolation of single objects or their parts in the scene that can be subsequently identified in an easier and more accurate way. Image segmentation has applications, such as object localization or recognition, data compression, tracking, image retrieval, or understanding. In recent years, a number of very inspiring and pioneering image segmentation algorithms have been developed, and these algorithms can be roughly classified into five major categories: thresholding, template matching, clustering, edge detection and region growing. These algorithms have been successful in many applications, but none of them are generally applicable to all images and different algorithms are usually not equally suitable for a particular application. The edge detection method is one of the widely used approaches to the problem of image segmentation. It is based on the detection of points with abrupt changes at gray levels.

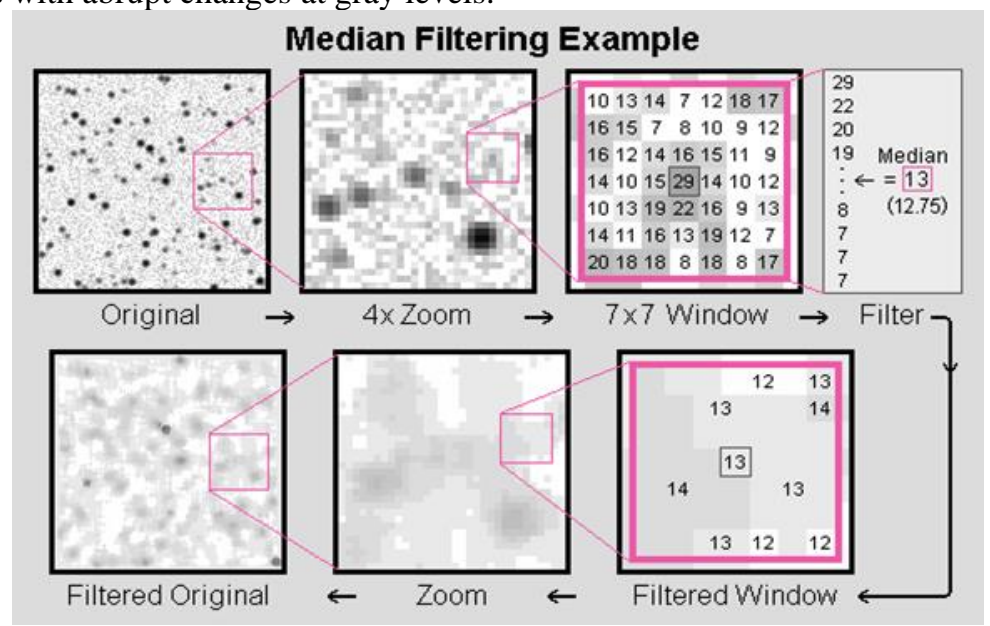

For recognition purpose, the iris part should be separated from the eye image. The iris is a ring shaped part in eye with pupil as inner boundary and sclera as outer boundary. Both the boundaries can approximately take as inner and outer circles of iris. For detecting the pupil and iris boundary, circular Hough transform is used. Canny edge detector is employing to generate an edge map, which finds edges where the grayscale intensity of the image changes the most. These areas are founded by determining gradients of the image. In order to make the circle detection process more efficient and accurate, the Hough transform for the iris/sclera boundary was performed first, then the Hough transform for the iris/pupil boundary was performed within the iris region, instead of the whole eye region, since the pupil is always within the iris region. After this process was complete, six parameters are stored, the radius and centre coordinates for both circles by the following equation.

$\mathrm{xc} 2+\mathrm{yc} 2-\mathrm{r} 2=0$

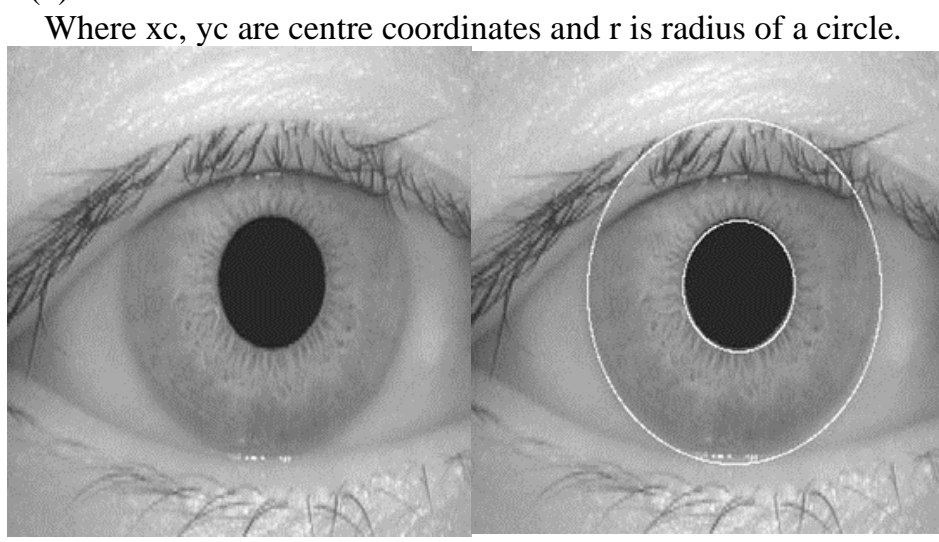

Original image

Localized image

Eyelids were isolated by first fitting a line to the upper and lower eyelids using the linear Hough transform. A second horizontal line is then drawn, which intersects with the first line at the iris edge that is closest to the pupil. This process is done for both upper and lower eyelids. The second horizontal line allows maximum isolation of eyelid regions.

\subsection{Image Normalization}


Normalization is done to convert circular iris pattern into rectangular shape. People have different sizes of iris. So, after iris localization process, the next stage is to transform the iris region so that it has fixed dimensions in order to allow comparisons. Factors which affect size of iris are intra- class variability i.e. illumination, varying of pose, noise and pollution, and inter-class variability i.e. person to person. During normalization, iris and pupil are non- concentric. These factors affect the result of iris matching. To get accurate result, localized iris is transformed from Cartesian to polar coordinate system. For transformation, remaps each point within the iris region to a pair of polar coordinates $(r, \Theta)$ where $r$ is on the interval $[0,1]$ and $\Theta$ is angle $[0,2 \Pi]$ as shown
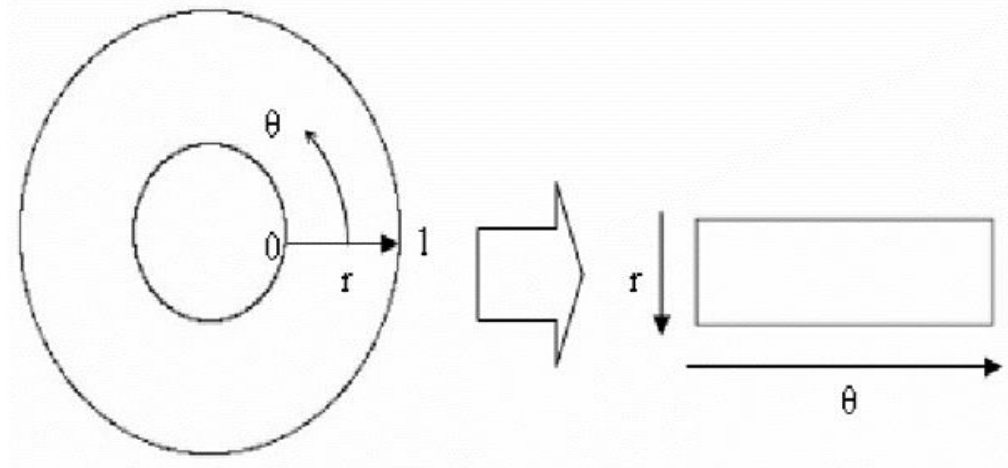

The remapping of the iris region from $(\mathrm{x}, \mathrm{y})$ Cartesian coordinates to the normalized non-concentric polar representation is modeled as
$\mathrm{I}(\mathrm{x}(\mathrm{r}, \Theta), \mathrm{y}(\mathrm{r}, \Theta)) \quad \mathrm{I}(\mathrm{r}, \Theta)$
With $x(r, \Theta)=(1-r) x(\Theta)+r x(\Theta)$
$\mathrm{y}(\mathrm{r}, \Theta)=(1-\mathrm{r}) \mathrm{yp}(\Theta)+\operatorname{ryl}(\Theta)$

Where $\mathrm{I}(\mathrm{x}, \mathrm{y})$ is the iris region image, $(\mathrm{x}, \mathrm{y})$ are the original Cartesian coordinates, $(\mathrm{r}, \Theta)$ are the corresponding normalized polar coordinates, and $\mathrm{xp}, \mathrm{yp}$ and $\mathrm{xl}, \mathrm{yl}$ are the coordinates of the pupil and iris boundaries along the $\Theta$ direction.

\subsection{ROI Extraction}

After normalization, the next step which comes into picture is ROI extraction. ROI extraction is nothing but cropping particular portion of normalized iris image according to IRDIO CHART.

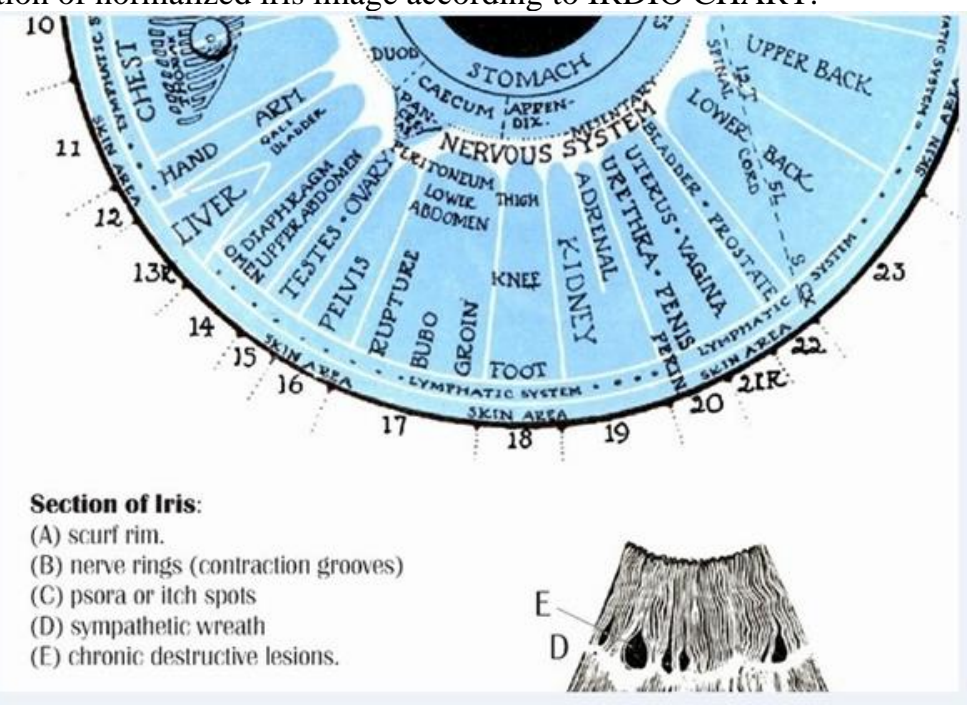

ROI- A region of interest is a portion of image that you want to filter or operate in some way. The toolbox supports a set of ROI objects that you can use to create ROIs of many shapes, such circles, ellipse, polygons, rectangle and hand drawn shapes.

Define an ROI by creating a binary mask, which is a binary image that is the same size as the image you want to process with pixels that defines the ROI set to 1 and all other pixels set to 0 . The real parts of filters are shorten to be volume zero and get illumination invariance. For each resulting bit, interpret the signs of the real and imaginary parts from quadrature image projection and through quantization assigned binary values: 1 for positive and 0 for negative projection values.

\subsection{Feature Extraction}

Feature extraction includes the features of the extracted region such as mean, variance, standard deviation etc. It will show all these features into the command window. According to the irido-chart, the region of interest can be identified. Principal Component Analysis (PCA) is used for extracting the features of region of interest. To apply the PCA on this ROI, matrix manipulation is necessary. Basically PCA assigns the specific weightage to the particular feature.

Feature indexes are:

$\mathrm{q}-$ The binary result of quality assessment. $0=$ bad quality $1=$ sufficient quality. 
ps - The binary result of pre-screening, where 1 indicates severe irishl abnormality and 0 its lack.

nma.a - nma.f - The results of micro aneurism detection. Each feature value stand for the number of micro aneurisms found at the confidence levels alpha $=0.5, \ldots, 1$, respectively.

nex.a - nex.h - contains the same information as nma.a - nma.f for exudates. However, as exudates are represented by a set of points rather than the number of pixels constructing the lesions, these features are normalized by dividing the number of lesions with the diameter of the ROI to compensate different image sizes.

dd - The euclidean distance of the center of the macula and the center of the optic disc to provide important information regarding the patient's condition. This feature is also normalized with the diameter of the ROI.

$\mathrm{dm}$-The diameter of the optic disc.

amfm - The binary result of the AM/FM-based classification.

class - Class label. 1 = contains signs of Diabetic Irish, $0=$ no signs of Diabetic Irish.

\subsection{SVM Classification}

SVM classification is the important part of the approach because the overall process depends upon the classification done through this algorithm. Classification in Machine Learning is the task of learning to distinguish points that belong to two or more categories in a dataset. In geometrical terms, associating a set of points to some category involves finding the best possible separation between these.

Support vector machine is another simple algorithm that every machine learning expert should have in his/her arsenal. Support vector machine is highly preferred by many as it produces significant accuracy with less computation power. Support Vector Machine, abbreviated as SVM can be used for both regression and classification tasks. But, it is widely used in classification objectives.

\section{Conclusion}

Diabetic Irish (DI) is human eye disease among people with diabetics which causes damage to irish of eye and may eventually lead to complete blindness. Detection of diabetic Irish in early stage is essential to avoid complete blindness. Our technique SVM gives more accurate results when compared with previous older techniques.

\section{References}

1. Li T, Meindert N, Reinhardt JM, Garvin MK, Abramoff MD (2013) Splat Feature ClassificationwithApplicationtoIr ishlHemorrhageDetectioninFundu sImages,IEEE Transactions on Medical Imaging, 32:364-375

2. Yau JW, Rogers SL, Kawasaki R, Lamoureux EL, Kowalski JW, Bek T, et al. Global prevalence and major risk factors of diabetic Irish. Diabetes Care.2012;35:556-64

3. BoserB ,Guyon I.G,Vapnik V., "A Training Algorithm for Optimal Margin Classifiers", Proc. Fifth Ann. Workshop Computational Learning Theory,pp. 144-152, 1992.

4. Mitchell, T. (1997). Machine Learning, McGraw Hill. ISBN 0- 07-042807-7.,McGrawHill, Inc. New York, NY, USA. Published on March 1, 1997 